\title{
Photosensitivity of the Structures on the $\mathrm{Cu}(\mathrm{In}, \mathrm{Ga})(\mathrm{S}, \mathrm{Se})_{2}$ Films Obtained by Thermal Treatment in the $S$ and Se Vapors
}

\author{
V. Yu. Rud' ${ }^{a}$, M. S. Tivanov ${ }^{b}$, Yu. V. Rud'c, V. F. Gremenok ${ }^{d}$, E. P. Zaretskaya ${ }^{d}$, \\ V. B. Zalesskii ${ }^{e}$, T. R. Leonova ${ }^{e}$, and P. I. Romanov ${ }^{e}$ \\ ${ }^{a}$ St. Petersburg State Polytechnical University, St. Petersburg, 195251 Russia \\ e-mail: rudvas@spbstu.ru \\ ${ }^{b}$ Belarussian State University, Minsk, 220050 Belarus \\ ${ }^{c}$ Ioffe Physicotechnical Institute, Russian Academy of Sciences, St. Petersburg, 194021 Russia \\ ${ }^{d}$ Joint Institute of Physics of Solid State and Semiconductors, Belarussian National Academy of Sciences, \\ Minsk, 220072 Belarus \\ ${ }^{e}$ Institute of Electronics, Belarussian National Academy of Sciences, Minsk, 220090 Belarus \\ Submitted November 7, 2006; accepted for publication February 28, 2007
}

\begin{abstract}
Using the method of simultaneous sulfurization and selenization of intermetallic $\mathrm{Cu}-\mathrm{In}-\mathrm{Ga}$ layers, single-phase thin films of the $\mathrm{Cu}(\mathrm{In}, \mathrm{Ga})(\mathrm{S}, \mathrm{Se})_{2}$ (CIGSS) alloys are obtained. On these films, rectifying photosensitive surface-barrier structures In/p-CIGSS are obtained by vacuum thermal evaporation of pure In. The photosensitivity spectra of the originally obtained structures are studied. The effect of the composition of the alloy films and illumination conditions on the photoelectric parameters of new structures In/p-CIGSS is studied. It is concluded that the obtained CIGSS films are promising for fabrication of high-efficiency thin-film photoconverters.
\end{abstract}

PACS numbers: 73.30.+y, 73.50.Pz, 81.40.Tv, 85.30.Hi

DOI: $10.1134 / \mathrm{S} 1063782607100089$

\section{INTRODUCTION}

Studies of ternary I-III-VI ${ }_{2}$ compounds and their alloys have led to the obtaining of high-efficiency radiation-resistant and inexpensive thin-film solar cells, which is the main problem of modern photoenergetics $[1,2]$. The development of $\mathrm{Cu}(\mathrm{In}, \mathrm{Ga}) \mathrm{Se}_{2}$ (CIGS) thin films has allowed researchers to obtain radiation-resistant solar cells with the efficiency of photoconversion higher than $19 \%$ and to open pilot production based on their moduli [3-6].

The main restriction in the technology of fabrication of CIGS films is the Ga segregation near the rear contact, which is determined by the difference of the reaction coefficients of formation of binary selenides [7]. As a result, this circumstance hinders the development of solar cells with the required gradient of the band gap over the film thickness. Researchers have succeeded in solving this problem by thermal treatment of the CIGS films in the $\mathrm{H}_{2} \mathrm{~S}$ atmosphere, which leads to substitution of Se by sulfur $\mathrm{Se} \longrightarrow \mathrm{S}$ and to the synthesis of a wide-gap $\mathrm{Cu}(\mathrm{In}, \mathrm{Ga})(\mathrm{S}, \mathrm{Se})_{2}$ (CIGSS) alloy in the nearsurface region of the film. Until now, the main technology of obtaining thin single-phase CIGSS films has been the method of vacuum coevaporation of $\mathrm{Cu}$, In, $\mathrm{Ga}, \mathrm{S}$, and Se. However, this method is unsuitable for the commercial production of thin CIGSS films because of its high cost and the complexity of control- ling the technology parameters [6]. At present, the possibilities of processes of thermal treatment of thin metal films $\mathrm{Cu}-\mathrm{In}-\mathrm{Ga}$ in the $\mathrm{H}_{2} \mathrm{~S}$ and $\mathrm{H}_{2} \mathrm{Se}$ atmospheres are studied as alternatives [6]. A serious obstacle for widespread application of this technology is the high toxicity of the $\mathrm{H}_{2} \mathrm{~S}$ and $\mathrm{H}_{2} \mathrm{Se}$ gases.

The main aim of this study was to develop the method of the synthesis of the CIGSS films of the required composition without the use of highly toxic gases and to fabricate the surface-barrier structures on the basis of these films in order to carry out the photoelectric monitoring of the development of high-efficiency solar cells.

\section{EXPERIMENTAL}

The synthesis of thin CIGSS films consisted of two technology stages, namely, the formation of the base $\mathrm{Cu}-\mathrm{In}-\mathrm{Ga}$ layers and their subsequent sulfurization and selenization in the S- and Se-containing inert atmosphere. At the first technology stage, the $\mathrm{Cu}-\mathrm{In}-\mathrm{Ga}$ base layers were deposited on glass substrates by the ion-plasma coevaporation in vacuum $\left(6.6 \times 10^{-4} \mathrm{~Pa}\right)$ at $100^{\circ} \mathrm{C}$. At the second stage, the CIGSS alloy was synthesized by the two-stage thermal treatment of the $\mathrm{Cu}-\mathrm{In}-\mathrm{Ga}$ intermetallic film in the $\mathrm{S}$ and Se vapors. The first stage of thermal treatment was carried out in the temperature range $220-240^{\circ} \mathrm{C}$. The second stage 

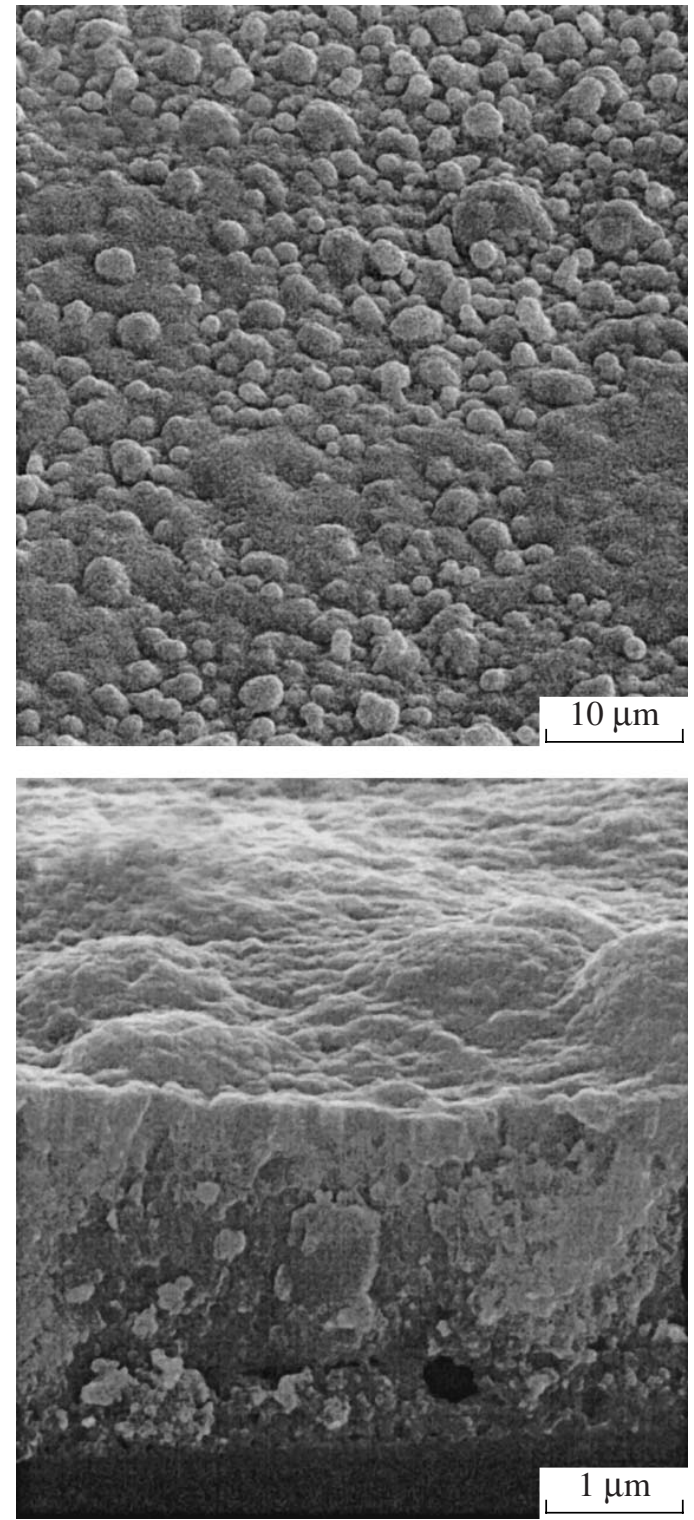

Fig. 1. Typical surface microprofile and transverse section of the CIGSS films under magnification indicated.

was carried out at the temperatures $500-550^{\circ} \mathrm{C}$, which are optimal. The partial pressure of chalcogen vapors ( $\mathrm{S}$ and $\mathrm{Se}$ ) was controlled by the temperature of their solid-phase sources. The reaction diffusion of $\mathrm{S}$ and $\mathrm{Se}$ was carried out in the inert gas medium $\left(\mathrm{N}_{2}\right)$ during the time providing the synthesis of the films of the CIGSS alloy of the required composition and crystal structure.

The structural properties and the phase composition of the obtained films were studied using the X-ray powder diffraction analysis. The phases were identified by comparison of experimentally determined interplanar spacings with the JCPDS tables. The main result of these investigations amounts to the conclusion that the used technology process provided the formation of thin films $\left(d_{l} \approx 2-2.5 \mu \mathrm{m}\right)$ with the crystal lattice of chal- copyrite, which is characteristic of the alloy studied, and the band gap $E_{g}=1.27-1.38 \mathrm{eV}$, which corresponds to the optimal value for solar converters [8].

The microprofile of the film surface, the film microstructure, and the transverse cleavage were studied by scanning electron microscopy using an $\mathrm{H}-800$ microscope (Hitachi, Japan) with the resolution $\sim 0.2 \mathrm{~nm}$.

The elemental composition of the obtained CIGSS films was studied by X-ray dispersion spectroscopy using a Stereoscan-360 system (Great Britain) equipped with an AH 1000 EDX spectrometer (Link Analytic, Great Britain) with the local resolution $\sim 1 \mu \mathrm{m}$ and sensitivity 0.1 at $\%$. The quantitative and qualitative analyses of the elemental composition over the depth of the grown films was carried out on a PHI-660 scanning Auger microprobe (Perkin Elmer, USA) with the local resolution $\sim 0.1 \mu \mathrm{m}$ and sensitivity 0.1 at $\%$ using an IMS-4F ion microprobe (Cameca). The quantitative analysis of the elemental composition of the grown films was carried out by the method of pure standards, where the current of the Auger electrons is corrected to the coefficient of elemental sensitivity.

\section{RESULTS AND DISCUSSION}

The study of the phase composition of the CIGSS layers, which were obtained under optimal synthetic conditions, showed that they contain only the structural phase of chalcopyrite, which corresponds to the $\mathrm{Cu}(\mathrm{In}, \mathrm{Ga})(\mathrm{S}, \mathrm{Se})_{2}$ alloy. The positional ordering of the atoms in the cationic sublattice is confirmed by the existence of superstructural reflections (101), (103), and splitting of doublets (116/312), (008/400), and (228/424).

The study of the microstructure of the synthesized layers shows that an increase in the recrystallization temperature (high-temperature stage) leads to an increase in the surface homogeneity without clearly pronounced segregation of separate phases. The crystallite growth takes place in the directions normal to the substrate plain. An increase in the temperature to the optimal value $\left(500-550^{\circ} \mathrm{C}\right)$ leads to the formation of CIGSS films with densely packed crystallites, which is an important condition for decreasing the diffusion of atoms of the buffer layer during fabrication of the barrier structures. This type of morphology provides the electric coupling of the grains, which is necessary for the effective operation of heterostructures based on polycrystalline films of these alloys. A typical microprofile and the transverse cleavage of the layers synthesized are shown in Fig. 1. The distribution of the components over the layer thickness, which is shown in Fig. 2, indicates that it is possible to obtain by this method thin films of CIGSS alloys with uniform distribution of components.

In Tables 1 and 2, the elemental composition and the properties of certain obtained films are given. The data of Table 1 and 2 confirm that this method is promising 
for the synthesis of thin films of CIGSS alloys of the specified composition with physical characteristics and the band gap $E_{g}^{\text {opt }} \approx 1.27-1.38 \mathrm{eV}$ necessary for highefficiency photoconversion of solar radiation.

It should also be noted that the variation in the concentration of elements in the grown films presented in Table 1 relatively weakly affects their resistivity, which equals $\rho \approx 10^{2} \Omega \mathrm{cm}$ at $T=300 \mathrm{~K}$, and does not lead to inversion of the conductivity type (all synthesized layers are of $p$-type conductivity). This circumstance allows us to assume that, in the films obtained, the ensemble of dominant lattice defects is not profoundly affected by the observed variation in the composition of the films (Table 1), and it can presumably be attributed to the existence of vacancies in the $\mathrm{Cu}$ sublattice. It is evident that all further studies dedicated to revealing the effect of increasing the concentration of the $\mathrm{Cu}$ atoms on the film resistance can be favorable to refinement of the nature of the dominant intrinsic lattice defects in the CIGSS films.

To study the photoelectric properties of the obtained CIGSS films, we fabricated surface-barrier structures. As the barrier material, we used pure In, which was deposited on the surface of the obtained $p$-CIGSS films by vacuum thermal evaporation similarly to $[9,10]$.

The measurements of the steady-state current-voltage characteristics of the obtained film structures In/p-CIGSS showed that they exhibit rectifying properties and the photovoltaic effect. The ratio between the forward and reverse currents for these films was at the level 2-5 at bias voltages $U \approx 2 \mathrm{~V}$, which is presumably associated with the imperfection of the In/p-CIGSS barrier interface and its periphery region. The conducting direction is realized at the negative polarity of the external bias at the barrier contact. On illumination of such structures, photovoltage emerges, which prevails if the barrier contact of these structures is illuminated. In this case, the photovoltage sign always corresponds to the rectification direction. The photovoltage sign for all obtained structures In $/ p$-CIGSS is independent of the photon energy, emission intensity, and location of the optical probe (diameter $\sim 0.3 \mathrm{~mm}$ ) on the photoreceiving surface of these structures. These regular features are the background for the conclusion that the found photovol-

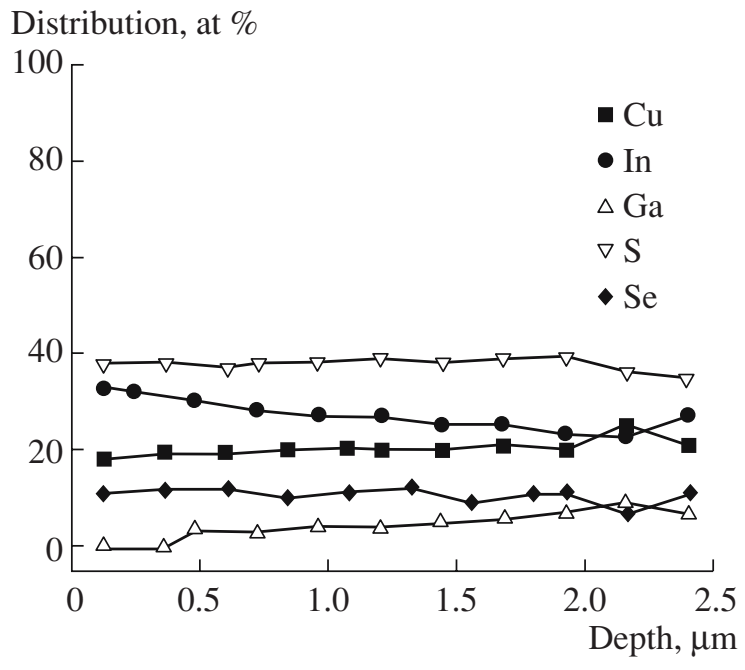

Fig. 2. Distribution of alloy components (sample no. $1 \mathrm{X} 213 \mathrm{~S} 2$ ) over the layer depth.

taic effect is determined by a single active region in the structure, which emerges on thermal deposition of the In film on the surface of the CIGSS alloy. The maximum voltaic photosensitivity $S_{U}^{\mathrm{m}}$ of the obtained structures (Table 2) varies in a rather wide range. However, we failed to reveal a clear interrelation of its value with the atomic composition of the films.

Figure 3 shows the spectral dependences of the relative quantum efficiency of photoconversion $\eta(\hbar \omega)$ for the series of the obtained structures. If the barrier-contact side of these structures is illuminated (Fig. 3, curves $1-4$ ), a broadband mode is always realized. The maximum value of the total width of the spectra $\eta(\hbar \omega)$ at their half-height $\delta_{1 / 2}$ (Table 2) for the structures under consideration was found to be fairly large (almost $2 \mathrm{eV}$ for the best structures), which indicates that a highly effective barrier is formed in these structures. The long wavelength exponential growth of photosensitivity for the structures under study starts at the photon energies $\hbar \omega \gtrsim 1 \mathrm{eV}$ (Fig. 3, curves 1-4). The spectral dependences of photosensitivity of the structures fabricated have a similar form. The exponential growth of photosensitivity of these structures is described by a fairly

Table 1. Composition of the CIGSS films

\begin{tabular}{l|c|c|c|c|c|c}
\hline \multirow{2}{*}{$\begin{array}{c}\text { Sample } \\
\text { number }\end{array}$} & \multicolumn{5}{c}{ Film compositions } \\
\cline { 2 - 7 } & $\begin{array}{c}\text { Molar fractions } \\
\text { of elements }\end{array}$ & $\mathrm{Cu}$ & $\mathrm{In}$ & $\mathrm{Ga}$ & $\mathrm{S}$ & $\mathrm{Se}$ \\
\cline { 2 - 7 } $1 \mathrm{X} 213 \mathrm{~S} 2$ & $\mathrm{Cu}_{0.82} \mathrm{In}_{1.22} \mathrm{Ga}_{0.04} \mathrm{~S}_{1.29} \mathrm{Se}_{0.63}$ & 20.49 & 30.45 & 0.95 & 32.27 & 15.83 \\
$1 \mathrm{X} 213 \mathrm{~S}$ & $\mathrm{Cu}_{0.73} \mathrm{In}_{1.18} \mathrm{Ga}_{0.09} \mathrm{~S}_{0.87} \mathrm{Se}_{1.13}$ & 18.34 & 29.44 & 2.19 & 21.79 & 28.24 \\
$1 \mathrm{X} 214 \mathrm{~S}$ & $\mathrm{Cu}_{0.85} \mathrm{In}_{1.19} \mathrm{Ga}_{0.09} \mathrm{~S}_{1.45} \mathrm{Se}_{0.43}$ & 21.17 & 29.71 & 2.15 & 36.25 & 10.71 \\
$2 \mathrm{X} 214 \mathrm{~S}$ & $\mathrm{Cu}_{0.79} \mathrm{In}_{1.22} \mathrm{Ga}_{0.02} \mathrm{~S}_{1.66} \mathrm{Se}_{0.31}$ & 19.68 & 30.53 & 0.54 & 41.55 & 7.70 \\
\hline
\end{tabular}


Table 2. Physical properties of the CIGSS films and the structures In/ $/ p$-CIGSS at $T=300 \mathrm{~K}$

\begin{tabular}{l|c|c|c|c|c|c|c|c|c}
\hline $\begin{array}{c}\text { Sample } \\
\text { number }\end{array}$ & $E_{g}^{\mathrm{opt}}, \mathrm{eV}$ & $d_{l}, \mu \mathrm{m}$ & $\rho, \Omega \mathrm{cm}$ & $\hbar \omega^{\mathrm{m}}, \mathrm{eV}$ & $\delta_{1 / 2}, \mathrm{eV}$ & $S_{U}^{\mathrm{m}}, \mathrm{V} / \mathrm{W}$ & $S, \mathrm{eV}^{-1}$ & $E_{g}^{\mathrm{d}}, \mathrm{eV}$ & $\varphi_{\mathrm{b}}, \mathrm{eV}$ \\
\hline $1 \mathrm{X} 213 \mathrm{~S} 2$ & $\sim 1.29$ & 2.2 & 88 & $1.6-2.2$ & 1.95 & 65 & 25 & 1.35 & 1.01 \\
$1 \mathrm{X} 213 \mathrm{~S}$ & $\sim 1.27$ & 2.2 & 150 & $1.6-2.5$ & 1.76 & 55 & 33 & 1.29 & 1.07 \\
$1 \mathrm{X} 214 \mathrm{~S}$ & $\sim 1.31$ & 2.5 & 150 & 1.5 & 1.13 & 25 & 23 & 1.31 & 1.12 \\
$2 \mathrm{X} 214 \mathrm{~S}$ & $\sim 1.38$ & 2.3 & 99 & 1.5 & 0.97 & 11 & 23 & 1.32 & 1.20 \\
\hline
\end{tabular}

large value of the slope $S=\delta(\ln \eta) / \delta(\hbar \omega) \approx 23-25 \mathrm{eV}^{-1}$, which allows us to attribute it to the direct band-to-band transitions. The exponential growth of $\eta$ in the obtained structures is completed in the vicinity of the photon energies 1.25-1.35 eV (Fig. 3, curves 1-4).

If the side of the thin semiconductor film in the obtained structures In/p-CIGSS is illuminated, the spectra $\eta(\hbar \omega)$ turn out to be of the narrow-band type

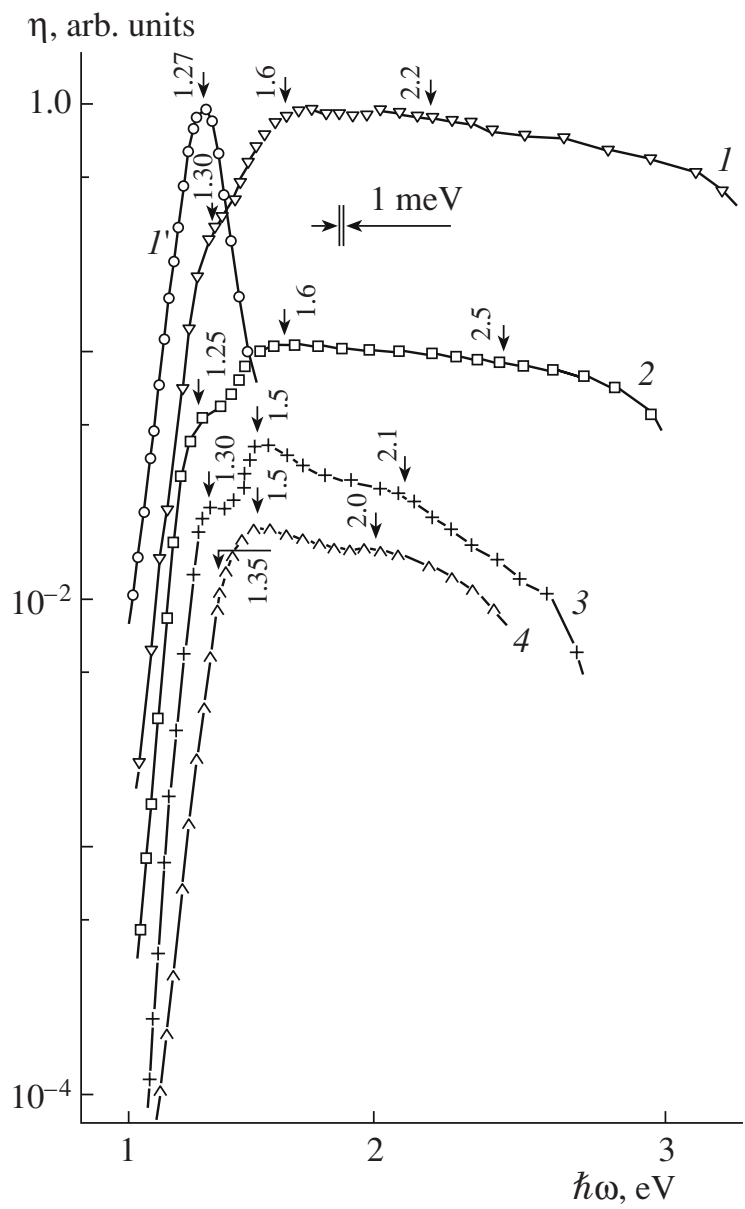

Fig. 3. Spectral dependences of relative quantum efficiency of photoconversion of the structures $\mathrm{In} / p-\mathrm{Cu}(\mathrm{In}, \mathrm{Ga})(\mathrm{S}, \mathrm{Se})_{2}$ at $T=300 \mathrm{~K}$. The sample numbers: $\left(1, I^{\prime}\right) 1 \mathrm{X} 213 \mathrm{~S} 2$, (2) $1 \mathrm{X} 213 \mathrm{~S}$, (3) $1 \mathrm{X} 214 \mathrm{~S}$, and (4) $2 \mathrm{X} 214 \mathrm{~S}$. (1-4) Illumination of the side of the barrier contact, and ( $\left.1^{\prime}\right)$ illumination of the side of the $\mathrm{Cu}(\mathrm{In}, \mathrm{Ga})(\mathrm{S}, \mathrm{Se})_{2}$ film.
(Fig. 3, curve $1^{\prime}$ ), and a clear short-wavelength falloff of photosensitivity emerges at $\hbar \omega>1.27 \mathrm{eV}$. This falloff is associated with an abrupt increase in the optical absorption and removal of the layer of photogenerated pairs from the active region. On this basis, the clear specificity of the $\eta(\hbar \omega)$ spectra in the region $\hbar \omega \approx$ $1.3 \mathrm{eV}$ in the case of illumination of the barrier side (Fig. 3, curves 1-4) can be attributed to the emergence of the direct band-to-band transitions in the alloy films, which agrees with the estimation of $E_{g}^{\text {opt }}$ from the absorption spectra of the same films (Table 2).

It is also seen from Fig. 3 (curves 1-4) that as the photon energy increases further, at $\hbar \omega>1.3 \mathrm{eV}$, the photosensitivity of the In/ $p$-CIGSS structures continues to increases and attains an absolute maximum at $\hbar \omega \approx$ $1.5-1.6 \mathrm{eV}$. It should be noted that with an increase in $\hbar \omega>1.6 \mathrm{eV}$, for the best structures in a wide range of photon energies $\hbar \omega^{\mathrm{m}}$ (Table 2), photosensitivity does not drop (Fig. 3, curves 1 and 2), while in the case of other structures at $\hbar \omega>1.5 \mathrm{eV}$ in the $\eta(\hbar \omega)$ spectra, a short-wavelength falloff appears. Consequently, we have reason to assume that the first measurements of photosensitivity spectra of the In/p-CIGSS structures shows the capacity of this barrier to suppress the effect of surface recombination. However, there is still no clear basis for attributing this fact only to variations in the atomic composition of the CIGSS alloys.

The analysis of the photosensitivity spectra of the obtained structures on the basis of the theory of the band-to-band absorption in semiconductors [11] allowed us to reveal the linear portions in dependences $(\eta \hbar \omega)^{2}=f(\hbar \omega)$, and, by their extrapolation to $\hbar \omega \longrightarrow 0$, to determine the values of the band gap in the electron spectrum of alloys for direct $E_{g}^{\mathrm{d}}$ band-to-band transitions (Fig. 4 and Table 2). It should be noted that these estimates of the band gap from optical $\left(E_{g}^{\mathrm{opt}}\right)$ and photoactive $\left(E_{g}^{\mathrm{d}}\right)$ absorption (Table 2$)$ turned out to be very close to each other and correspond to the known condition for obtaining the maximum photoconversion efficiency [8].

It also follows from Fig. 4 that, for the barriers $\mathrm{In} / p$ CIGSS, the long-wavelength part of photosensitivity corresponds to the Fowler law, which indicates the photoemission of nonequilibrium charge carriers $[11,12]$. Extrapolation of the dependence $(\eta \hbar \omega)^{1 / 2} \longrightarrow 0$ 


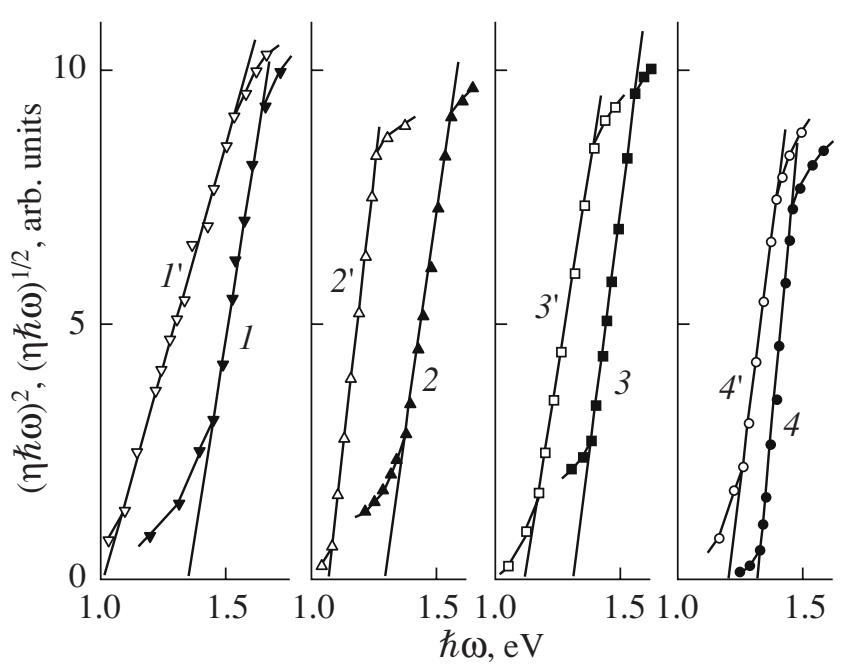

Fig. 4. Dependences $(\eta \hbar \omega)^{2}=f(\hbar \omega)$ (curves 1-4) and $(\eta \hbar \omega)^{1 / 2}=f(\hbar \omega)$ (curves $\left.l^{\prime}-4 '\right)$. The numbers of the samples: (1, 1') 1X213S2, (2, 2') 1X213S, (3, 3') 1X214S, and $\left(4,4^{\prime}\right) 2 \mathrm{X} 214 \mathrm{~S}$.

allowed us to estimate the height of the energy barrier $\varphi_{b}$, which appears at the In contact with the alloy film (Fig. 4, Table 2). One can see that the obtained values of $\varphi_{\mathrm{b}}$ are in the range $\varphi_{\mathrm{b}} \approx 1-1.2 \mathrm{eV}$, while the observed variations are apparently associated with the variation in the composition of the GIGSS alloy.

\section{CONCLUSIONS}

The basis of the technology of obtaining the films of the CIGSS alloys by the method of simultaneous sulfurization/selenization of intermetallic $\mathrm{Cu}-\mathrm{In}-\mathrm{Ga}$ layers without the use of highly toxic compounds is developed. The single-phase films of the CIGSS solutions $\sim 2.5 \mu \mathrm{m}$ thick with the uniform distribution of the components over the layer thickness are synthesized, and new structures In/ $p$-CIGSS are obtained on the basis of these films. The completed studies show the potential for controlling the properties of photosensitive structures by controlling the parameters of the technological process.
The suggested technology is suitable for the commercial production of thin large-area CIGSS films, which can be used in high-efficiency thin-film solar cells.

\section{ACKNOWLEDGMENTS}

This study was supported by the State Complex Program of Scientific Investigations of Republic Belarus "Energobezopasnost' (Energy Safety) 2006-2010," and partially supported by the Foundation for Basic Research of Belarus and by the Program of the Russian Academy of Sciences "New Principles of Energy Conversion in Semiconductor Structures."

\section{REFERENCES}

1. L. L. Kazmerski, Renewable Sustainable Energy Rev. 1 (1, 2), 71 (1997).

2. A. Goetzberger, C. Hebling, and H. W. Schock, Mater. Sci. Eng. R 40, 1 (2003).

3. K. Ramanathan, M. A. Contreras, C. L. Perkins, et al., Prog. Photovoltaics: Res. Appl. 11, 225 (2003).

4. M. Powalla and B. Dimmler, Sol. Energy Mater. Sol. Cells 75, 27 (2003).

5. J. Palm, V. Probst, and F. H. Karg, Sol. Energy 77, 757 (2004).

6. V. Alberts, J. Titus, and R. W. Birkmire, Thin Solid Films 451-452, 207 (2004).

7. I. M. Kotschau, M. Turcu, U. Rau, and H. W. Schock, Mater. Res. Soc. Symp. Proc. 668, H4.5.1 (2001).

8. J. I. Pankove, Optical Processes in Semiconductors (Prentice-Hall, Englewood Cliffs, N.J., 1971; Mir, Moscow, 1973).

9. I. V. Bodnar', T. A. Kushner, V. Yu. Rud', and Yu. V. Rud', Zh. Prikl. Spektrosk. 69, 520 (2002).

10. V. Yu. Rud, Yu. V. Rud, V. F. Gremenok, et al., Solid State Phenom. 67-68, 415 (1999).

11. S. M. Sze, Physics of Semiconductor Devices, 2nd ed. (Wiley, New York, 1981; Mir, Moscow, 1984).

12. A. G. Milns and J. J. Feucht, Heterojunctions and Metal-Semiconductor Junctions (Academic, New York, 1972; Mir, Moscow, 1975).

Translated by N. Korovin 